\title{
Adverse drug reactions in primary care: a scoping review
}

H. Khali ${ }^{1,2^{*}}$ and C. Huang ${ }^{2}$

\begin{abstract}
Background: Medication-related adverse events, or adverse drug reactions (ADRs) are harmful events caused by medication. ADRs could have profound effects on the patients' quality of life, as well as creating an increased burden on the healthcare system. ADRs are one of the rising causes of morbidity and mortality internationally, and will continue to be a significant public health issue with the increased complexity in medication, to treat various diseases in an aging society. This scoping review aims to provide a detailed map of the most common adverse drug reactions experienced in primary healthcare setting, the drug classes that are most commonly associated with different levels/types of adverse drug reactions, causes of ADRs, their prevalence and consequences of experiencing ADRs.
\end{abstract}

Methods: We systematically reviewed electronic databases Ovid MEDLINE, Embase, CINAHL Plus, Cochrane Central Register of Controlled Trials, PsycINFO and Scopus. In addition, the National Patient Safety Foundation Bibliography and the Agency for Health Care Research and Quality and Patient Safety Net Bibliography were searched. Studies published from 1990 onwards until December 7, 2018 were included as the incidence of reporting drug reactions were not prevalent before 1990. We only include studies published in English.

Results: The final search yielded a total of 19 citations for inclusion published over a 15-year period that primarily focused on investigating the different types of adverse drug reactions in primary healthcare. The most causes of adverse events were related to drug related and allergies. Idiosyncratic adverse reactions were not very commonly reported. The most common adverse drug reactions reported in the studies included in this review were those that are associated with the central nervous system, gastrointestinal system and cardiovascular system. Several classes of medications were reported to be associated with adverse events.

Conclusion: This scoping review identified that the most causes of ADRs were drug related and due to allergies. Idiosyncratic adverse reactions were not very commonly reported in the literature. This is mainly because it is hard to predict and these reactions are not associated with drug doses or routes of administration. The most common ADRs reported in the studies included in this review were those that are associated with the central nervous system, gastrointestinal system and cardiovascular system. Several classes of medications were reported to be associated with ADRs.

\section{Background}

Medication-related adverse events, or adverse drug reactions (ADRs) are harmful events caused by medication. Adverse drug reactions (ADRs) are defined by the World Health Organization (WHO) as "a response to a medication that is noxious and unintended used in man to

\footnotetext{
* Correspondence: h.khalil@latrobe.edu.au

${ }^{1}$ School of Psychology and Public Health, Department of Public Health, Latrobe University, Collins Street., Melbourne, Vic 3000, Australia

${ }^{2}$ Monash University, Clayton, Vic 3825, Australia
}

treat" [1]. ADRs could be a result of a preventable medication error, resulting in a side-effect as a result of medication administration, or an unforeseen error such as an allergic reaction $[2,3]$.

ADRs could have profound effects on the patients' quality of life, as well as creating an increased burden on the healthcare system. ADRs are one of the rising causes of morbidity and mortality internationally, and will continue to be a significant public health issue with the increased complexity in medication, to treat various diseases in an aging society. A recent study showed that 
ADRs accounted for approximately 3.5\% of hospital admission [4,5]. Furthermore, ADRs were the cause of $\sim 197,000$ deaths in Europe annually [1].

The causes and nature of adverse drug events are often complex and multifactorial. The types of adverse reactions are classified into the following categories: dose/drug related, allergic or idiosyncratic reactions. Dose-related and drug related adverse drug reactions are usually related to the dose of the medication and are usually predictable but sometimes unavoidable [6-9]. It is highly dependent on the patient's sensitivity to the drug and combinations of medication used. It generally does not lead to severe ADR but is relatively common. An allergic drug reaction is when the patients develops an inappropriate reaction to the medication, which mostly could be avoided with a skin test prior to or through effective consultation and communication between primary care facilities and patients. An idiosyncratic adverse drug reaction is a type that is not widely understood and its severity is often quite unpredictable. This affects the fewer people and the reason for the adverse reaction may be genetically predetermined [9].

ADRs have become a significant problem in patients who are on multiple medications such as the elderly. A study has reported that as high as $75 \%$ of all aged care residents had medication discrepancies after the transition from hospital to primary care setting [6].

Most of the adverse medication events are associated with prescription errors in general practice [7]. Medication errors in general practice had a prevalence rate of $5 \%$ in England according to a large retrospective case review study [8]. With the incorporation of technology in healthcare system, the implementation of computerized prescribing systems also has a range of medication error rates that may lead to mild or severe adverse drug events $[10,11]$.

Another cause of adverse events is the off -label use of uncommon medications in children and patients. Off label prescribing is the process of prescribing of medications to non-approved indications by organizations such the Therapeutic Goods Administration of Australia or the Food and Drug Administration agency in the United States. Medication error or dosage error can occur in these circumstances due to the lack of evidence to support their use in non-approved conditions [12-14].

To date, there is limited data and evidence on the epidemiology of ADRs. After a preliminary search of literature, (i.e. The Cochrane Library, JBI Database of Systematic Reviews and Implementation Reports, Ovid MEDLINE) there are no systematic reviews, metaanalysis or scoping reviews that provide a comprehensive overview of the types of adverse events in primary care. Most of the studies available were relatively small, and often confined to individual units. Alternately, most of the current reviews focused on the occurrence of medication errors, specific interventions to reduce medication errors and medication management [15-22]. While there are several reviews on medication programs focusing on the effect of medication errors and effectiveness of interventions, they do not describe the types of adverse events [21, 22]. The review by Khalil et al., 2017 examined the effectiveness of various types of medication safety interventions to reduce mortality, emergency visits and hospital admissions. The authors found little evidence to support the benefits of organizational, professional and structural interventions addressing medication errors due to the heterogeneity of the included studies [21]. .Assiri et al., 2018 examined the prevalence of medications errors and adverse events associated with errors and risk factors associated with them. They found inconsistencies in the definitions of medications errors, methodologies used to detect adverse events and different outcome measures.

Therefore, this review sought to address the type of ADRs, the major drug classes associated with the reactions, causes of ADRs, their prevalence as well as consequences of experiencing ADRs to reduce the risk of adverse drug events in primary care. This will enable clinicians to be more informed of the adverse events and which class of drugs are associated with them. Targeted educational interventions addressing these gaps have the potential to improve patient safety. This scoping review will also be useful for researchers and healthcare providers as well as policy makers in the development of interventions to reduce adverse drug reactions in today's primary care.

\section{Inclusion criteria \\ Participants}

This review considered participants of any age and any condition treated and/or managed from any primary care services.

\section{Concept}

The concept of interest for the scoping review was the type of adverse drug reactions experienced by patients and the classes of medications associated with these adverse drug events.

\section{Context}

The context of the review was the primary care setting. These include; primary health care organizations, general practitioner clinics, pharmacies, outpatient clinics and any other clinics that do not classify patients as inpatients. We only excluded hospital patients.

\section{Types of studies}

This scoping review considered quantitative study designs including experimental, descriptive and observational studies reporting any quantitative data that can be 
included in the review. Qualitative studies were not considered in this review as the data extracted were not eligible for inclusion as mentioned in the scoping protocol [23]. Due to time constraints, only data published in English were considered for the review. No gray literature was searched as we are interested in studies that are published in peer reviewed journals based on scientific methods that use evidence to develop conclusions.

\section{Search strategy}

The search strategy aimed to identify studies published from 1990 to 2018. A three-step search strategy was utilized in this review. An initial limited search of Ovid MEDLINE, JBI Database of Systematic Reviews and Implementation Reports and Cochrane Central Register of Controlled Trials was undertaken followed by analysis of the text words contained in the title and abstract and of the index terms used to describe the article. A second search using all identified keywords and index terms was undertaken across all included databases. The following databases were searched on December 7, 2018: Ovid MEDLINE, Embase, CINAHL Plus, Cochrane Central Register of Controlled Trials, PsycINFO and Scopus. The search strategy of all the databases followed the same strategy shown in Appendix I. In addition, the National Patient Safety Foundation Bibliography and the Agency for Health Care Research and Quality and Patient Safety Net Bibliography were searched. Studies published from 1990 onwards until December 7, 2018 were included as the incidence of reporting drug reactions were not prevalent before 1990. The reference list of all identified reports and articles were searched for additional studies. The following keywords were used: patient safety, adverse events, harmful incidents, primary care, aged care, ambulatory care, general practice and home healthcare. These were used along with a comprehensive list of variations of these key terms.

\section{Method}

\section{Data extraction}

Relevant data were extracted from the included studies to address the review question using the methodology outlined by Peters et al. [24, 25] The data extracted followed the template developed in the protocol [23]. .Please refer to the search strategy published in the protocol [23].

The data extracted included the following: author(s), year of publication, origin/country of origin (where the study was published or conducted), aims/purpose, study population, methodology/methods, context, types of adverse drug reactions experienced by patients and the classes of medications associated with them as shown in Tables 1 and 2.

\section{Results}

The database search yielded a total of 4462 citations after duplicates were removed. The titles and abstracts for these 4462 citations were screened and 4426 had irrelevant titles and abstracts and therefore excluded. The remaining $36 \mathrm{pa}-$ pers were selected for further assessment of the full-text assessment. Of these, 17 were excluded due to having: an irrelevant setting that is not primary care, irrelevant interventions that were only addressing medication errors instead of reporting on drug related adverse events and describing only qualitative aspects of medication safety. The final search yielded a total of 19 citations for inclusion in this review, with two abstracts and 17 full papers [26-44]. A protocol detailing the methodology for the current review was followed [23]. A PRISMA flowchart showing the study selection at each stage is detailed in Fig. 1. Tables 1 and 2 detail the study characteristics and the outcomes.

\section{Studies characteristics}

\section{Authors and year of publication/country of origin}

The included studies were published between 2003 and 2018. Most of the studies included were undertaken in developed countries such as USA, Germany, Sweden. Details of the studies country of origin are presented in Table 1.

\section{Study population}

The population size for the included studies ranged from 2842 to $33,891,339$ patients from across databases searched for this study. The types of participants included elderly residents, cancer patients, epileptic patients, multidrug-resistant $\mathrm{TB}$ patients, pediatrics and general adult patients.

\section{Method}

The types of studies included mainly observational cohort studies, retrospective case reviews and health record reviews.

\section{Context}

All studies were conducted in primary care settings. Eleven were set in primary care centers, 12 were set in outpatient clinics, two were set in general practice clinics, one was set in a residential nursing facility, and one was set in home care.

\section{Type of adverse drug reactions (context)}

The types of ADRs are categorized into three groups: drug related, allergic reaction and idiosyncratic reactions. The majority of studies have addressed drug related adverse reactions followed by allergic reactions. Only four studies addressed idiosyncratic reactions [29, 33, 38, 42]. ADRs were classified either by systems (Central nervous systems, cardiovascular events, etc. ...) or by 


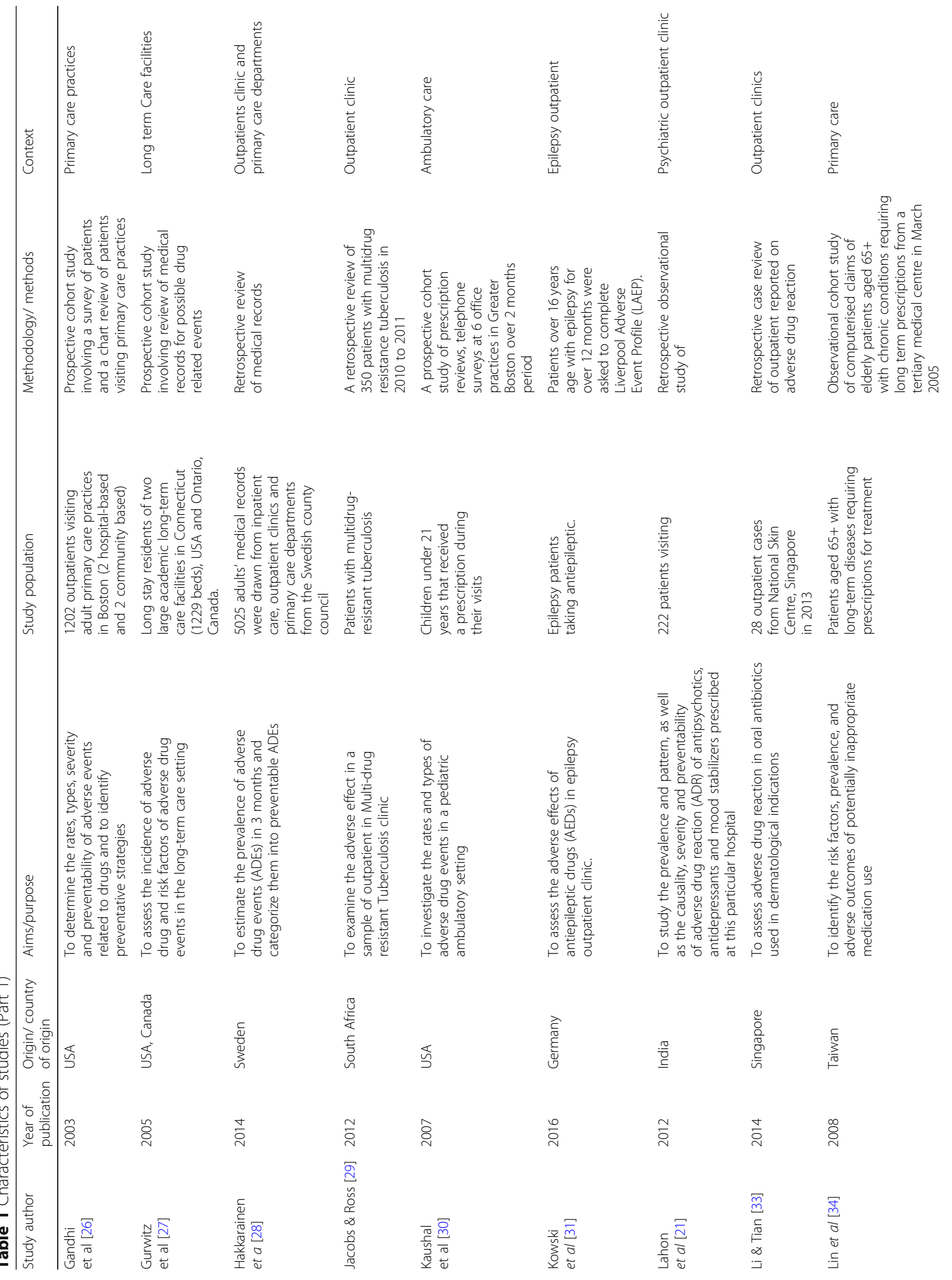




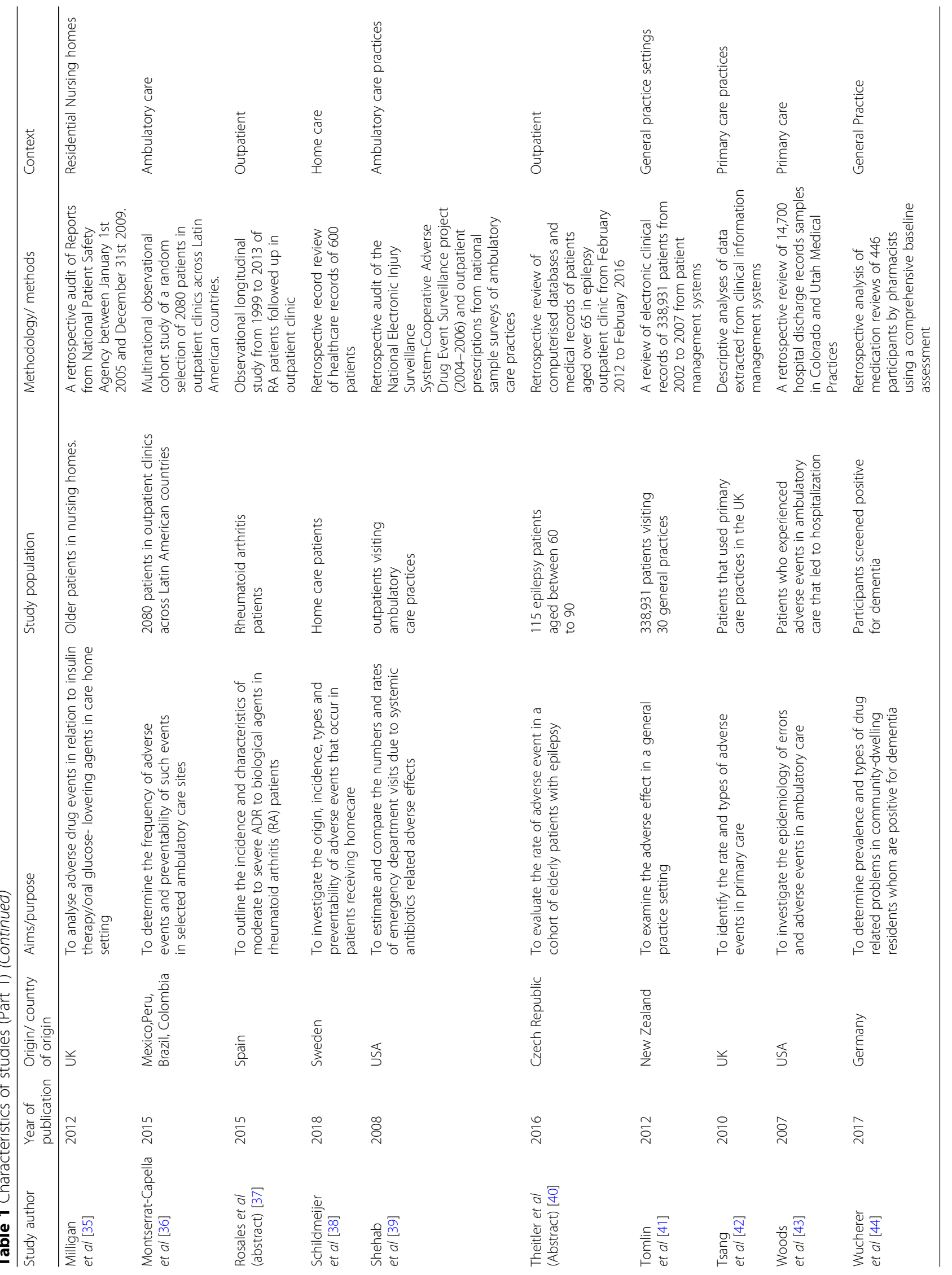




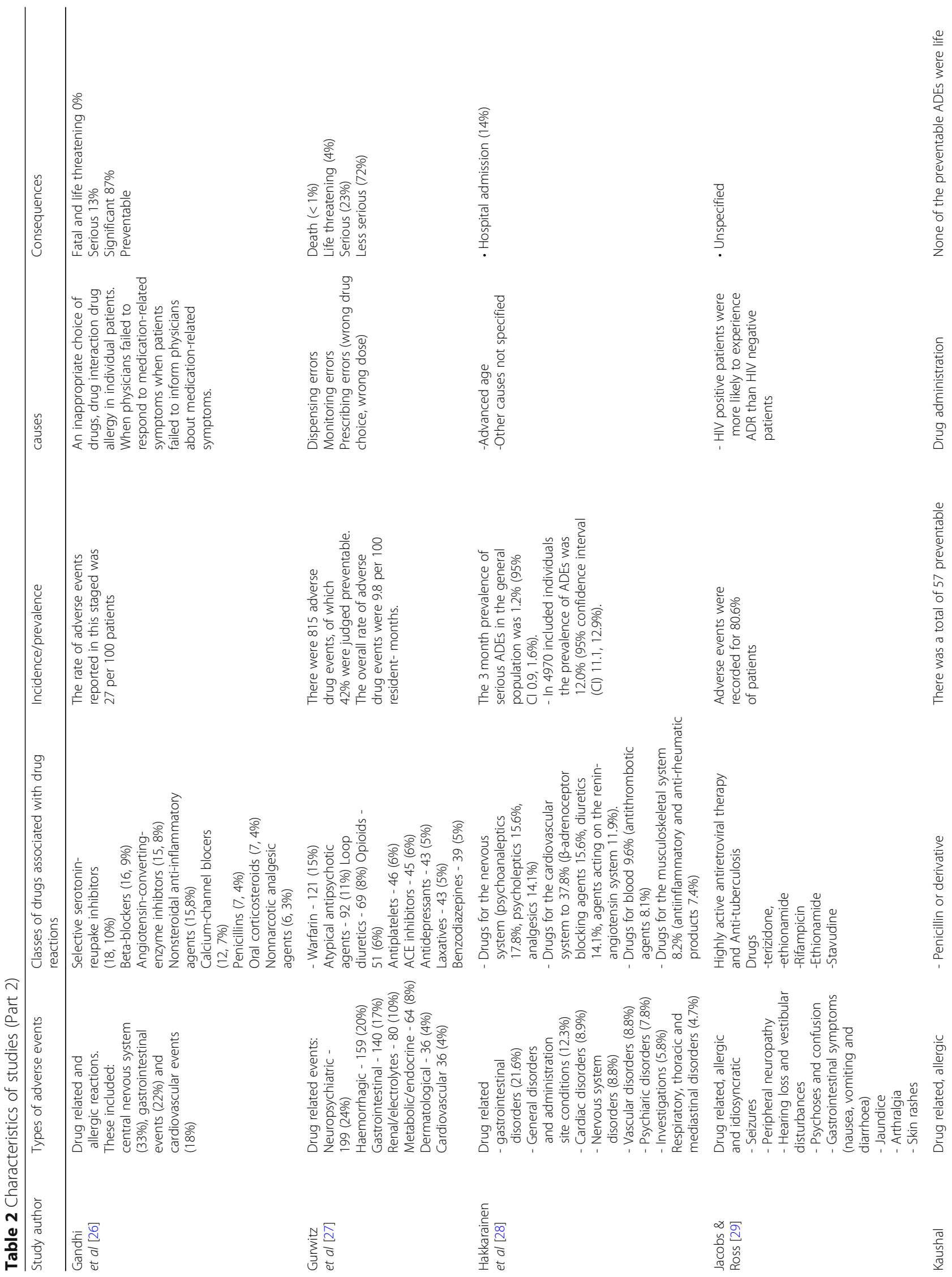




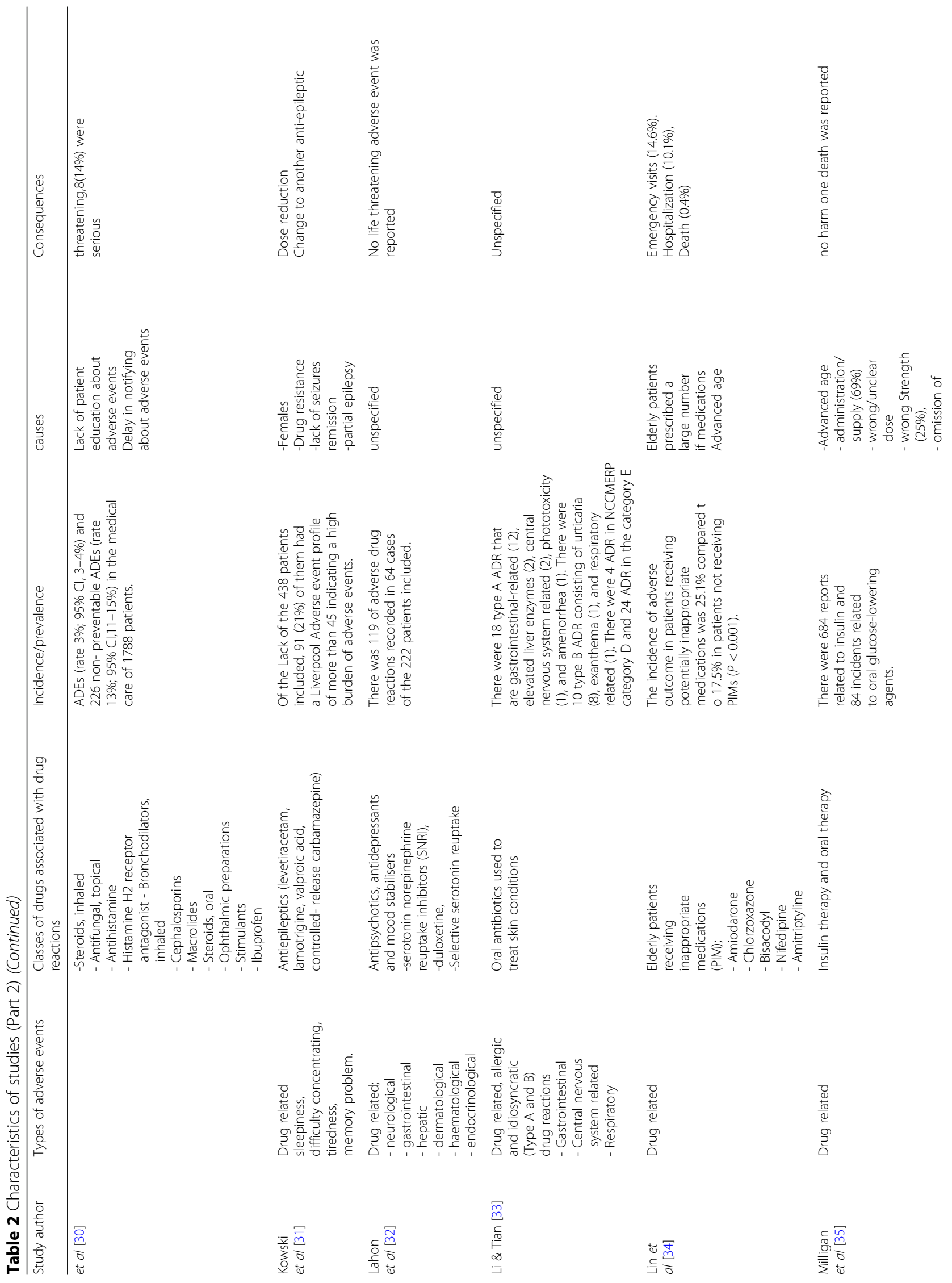




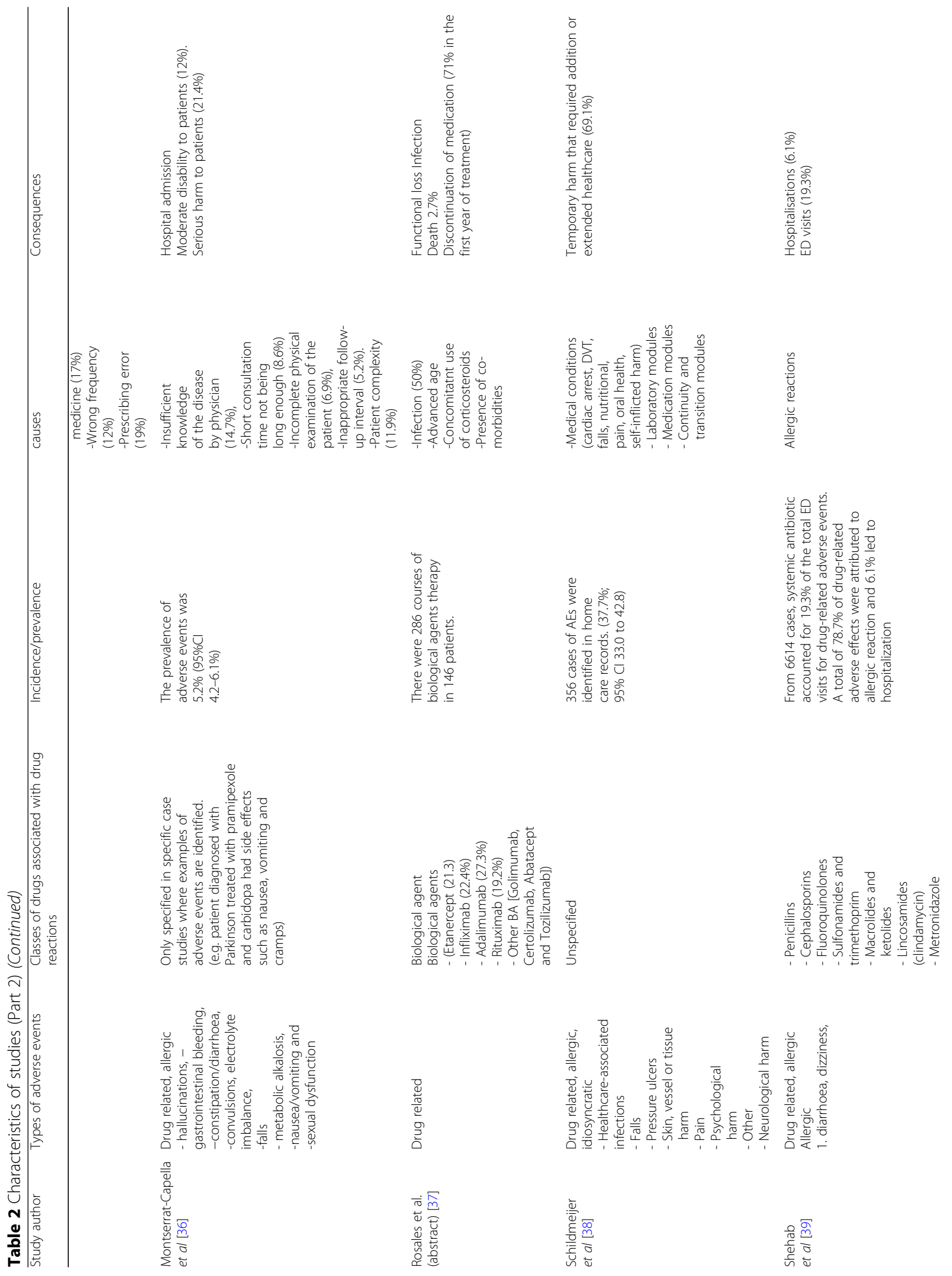




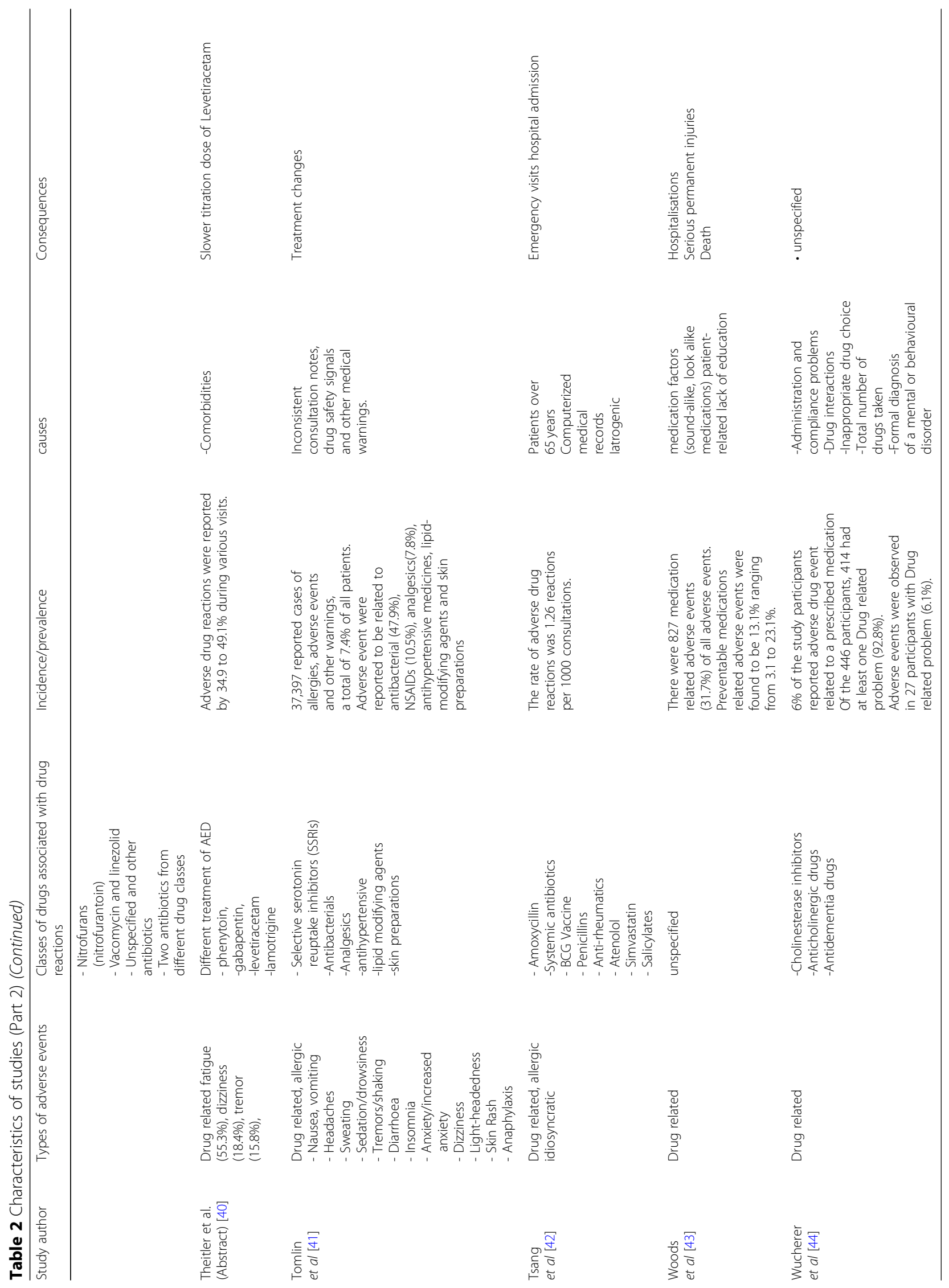




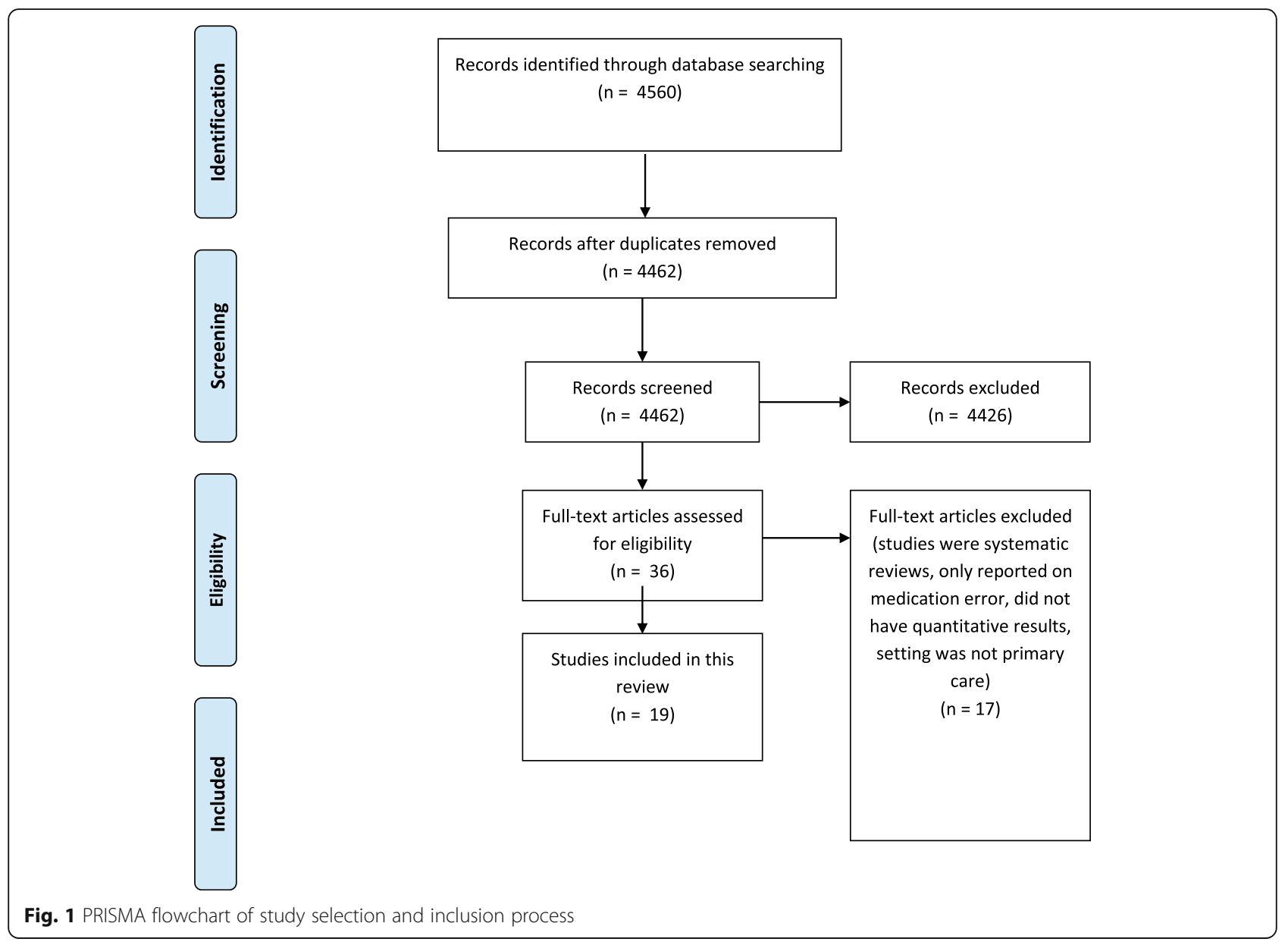

adverse reactions (ie. seizures, hearing loss, etc.). The frequency of ADRs reported were not included in all the studies. The most frequent ADR was related fatigue (55\%) followed by dizziness (18.4\%) and tremor (15.8\%) [40]. The body system that was associated with the most ADRs reported was the central nervous system followed by gastrointestinal and cardiovascular systems $[26,28]$.

\section{Classes associated with ADRs (context)}

A total of nine studies out of the 19 included studies addressed specific classes of medications such as; antituberculosis drugs [29], anti-epileptics [31, 40], antipsychotics, antidepressants and mood stabilisers [32], antibiotics [33, 39], insulin and oral diabetic medications [35], biologicals [37], and anticholinergic drugs including dementia medications [44]. The remainder of the studies covered other classes of medications such as beta blockers, antiplatelets, analgesics, benzodiazepines, musculoskeletal drugs, stimulants, lipid modifying agents, selective serotonin reuptake inhibitors and skin preparations. The classes of drugs that were associated with the highest ADRs reported in the included studies were drugs used for the cardiovascular system (beta-adrenergic blocking agents, diuretics, ACE inhibitors) warfarin, antipsychotic agents and opioids analgesics [26-28].

\section{ADRs incidences}

There was no standardized reporting of the prevalence data in the included studies. Prevalence data varied from simple calculations of the frequency of ADR in the study populations to an estimated number of adverse events per 100 patients, 100 residents' month, number of reactions per 1000 consultations [26. 28, 42]. Overall, the incidence of ADR reported in the studies ranged between $6 \%$ and up to $80 \%$ in some cases $[29,44]$.

\section{Causes of ADRs reported}

The causes of ADR varied between the studies. However, the majority of the authors cited patient factors as the cause of ADRs such as advanced age, lack of patients' education and patients' comorbidities [4, 26, 28-31, 34$38,40,42]$.

Some studies mentioned drug related causes such as prescribers' errors, inconsistent consultation notes, incomplete physical examination, inappropriate follow up and monitoring errors as causes of ADRs [26, 27, 35, 36, 38, 41, 44]. 
Drug specific causes such as drug administration, dispensing errors, drug interactions and look alike medications were also mentioned by three studies [27, 30, 35, 43].Allergic reactions were cited as the cause of ADRs in one study by Shehab et al. [39] Iatrogenic causes was also cited by one study amongst other causes [42]. Two studies did not specify any causes for the reported ADR [32, 33].

\section{Consequences of ADRs}

The consequences of the ADRs reported in the included studies ranged from medication cessation to death in some cases. Hospitalizations were reported in seven studies [28, 34, 36, 38, 39, 42, 43]. Death was reported in six studies $[26,27,34,35,37,43]$.

\section{Discussion}

This scoping review identified that the most causes of ADRs were drug related and due to allergies. Idiosyncratic adverse reactions were not very commonly reported in the literature. This is mainly because it is hard to predict and these reactions are not associated with drug doses or routes of administration [45]. The most common ADRs reported in the studies included in this review were those that are associated with the central nervous system, gastrointestinal system and cardiovascular system. Several classes of medications were reported to be associated with ADRs.

The prevalence of ADRs varied significantly between the studies, reasons for this variation include study designs, characteristics of participants and setting of the study and study length. These results are consistent with a similar review of observational studies [46]. Studies addressing children are also underrepresented in this review. We only found one study that met our inclusion criteria where the authors investigated the rates and types of ADRs in a pediatric ambulatory setting [30].

The causes of ADRs in this review were found to be multifactorial. These included: patient related factors such as co-morbidities, drug interactions, older age, provider characteristics such as monitoring errors, administration errors, incorrect drug selection and drug specific such as allergies or idiosyncratic reactions. Therefore, it is reasonable to predict their occurrences in primary care settings. This is in line with other findings from similar reviews [47].

Hospitalization and mortality were reported in less than half the studies included. Hospitalizations due to ADRs ranged between 6 to $14 \%$ which is comparable to other systematic reviews [48-50]. Mortality rates ranged between 0.4 to $2.7 \%$ in the studies included in this review. Under reporting of adverse events have been cited in the literature [51]. This may have been due to several factors including barriers to reporting within each organization, clinicians' reluctance to report to avoid punishment or blame [52]. Other barriers could be lack of knowledge about adverse events and whether they are related to the actual condition or the medications $[51,52]$. Complacency and other personal factors related to clinicians such as fear of being ridiculed of reporting merely suspected ADRs and fatigue were also reported [53].

Health care professionals are encouraged to be aware of the most commonly classes of drugs associated with ADRs such as cardiovascular drugs, antipsychotics and opioids as found in these studies. Targeted educational interventions to address underreporting of ADRs is essential to improve public health safety. There are many reasons for underreporting ADRs especially in children is paramount to improve patient safety. Our review highlighted the limited number of studies reporting ADRs in children.

Personal medicine is the approach where health professionals tailor specific treatments for individual patients to optimize outcome and reducing ADRs. As today's society moves towards personalized medicine, by understanding the causes and nature of ADRs, healthcare providers can extend the benefits and limit adversity on a personal level. By understanding the population and the groups of medications that are particularly susceptible to ADRs, health professionals can make better medication selections and improved dosing for the specific populations [54]. Extensions into research of pharmacogenomics will also improve the understanding of ADRs. Understanding the impact of genetics on drug effects have the potential to predict ADRs.

\section{Limitations of the review}

This review has a few limitations. There was also limited data from the included studies in regard to the ADRs and classes of medications associated with them. Furthermore, most of the studies were undertaken in developed countries. Applying these results to other countries might not be relevant due to the various systems in reporting ADRs. This is in addition to the limitations in the included studies such as small sample sizes, heterogeneous populations, variations in outcome measures.

\section{Conclusion}

This scoping review identified that the most causes of ADRs were drug related and due to allergies. Idiosyncratic adverse reactions were not very commonly reported in the literature.

This is mainly because it is hard to predict and these reactions are not associated with drug doses or routes of administration. The most common ADRs reported in the studies included in this review were those that are associated with the central nervous system, gastrointestinal system and cardiovascular system. Several classes of medications were reported to be associated with ADRs. 


\section{Abbreviations}

ADRs: Adverse drug reactions; ACE inhibitors: Angiotensin converting enzyme inhibitors; PRISMA: Preferred Reporting Items for Systematic Reviews and Meta-Analyses; TB: tuberculosis

\section{Acknowledgements}

The authors would like to Thank Diana Gee for her initial help with data searching and endnote.

\section{Authors' contributions}

HK designed the study, undertook the databases searches, drafted the manuscript, $\mathrm{CH}$ undertook the data analysis and data extraction. Both authors read and approved the final manuscript.

\section{Funding}

None.

\section{Availability of data and materials}

Not applicable.

\section{Ethics approval and consent to participate}

Not applicable.

\section{Consent for publication}

The authors consent to publish the manuscript.

\section{Competing interests}

The authors declare that they have no competing interests.

\section{Received: 9 May 2019 Accepted: 16 October 2019}

\section{10.}

\section{References}

1. Hadi MA, Neoh CF, Zin RM, Elrggal ME, Cheema E. Pharmacovigilance: pharmacists' perspective on spontaneous adverse drug reaction reporting. Integrated Pharmacy Res Pract. 2017;6:91-8.

2. Nivya K, Sri Sai Kiran V, Ragoo N, Jayaprakash B, Sonal Sekhar M. Systemic review on drug related hospital admissions - a pubmed based search. Saudi Pharmaceut J. 2015;23(1):1-8.

3. Jokanovic N, Wang KN, Dooley MJ, Lalic S, Tan EC, Kirkpatrick CM, Bell JS. Prioritizing interventions to manage polypharmacy in Australian aged care facilities. Res Soc Adm Pharm. 2017;13(3):564-74.

4. Aagaard L, Strandell J, Melskens L, Petersen PS, Hansen EH. Global patterns of adverse drug reactions over a decade. Drug Safety. 2012;35(12):1171-82.

5. Stausberg J. International prevalence of adverse drug events in hospitals: an analysis of routine data from England, Germany, and the USA. BMC Health Serv Res. 2014;14:125.

6. Marcum ZA, Handler SM, Boyce R, Gellad W, Hanlon JT. Medication misadventures in the elderly: a year in review. Am J Geriatr Pharmacother. 2010;8(1):77-83.

7. Coxon J, Rees J. Avoiding medical errors in general practice. Trends Urol Men's Health. 2015;6(4):13-7.

8. Avery AJ, Ghaleb M, Barber N, Dean Franklin B, Armstrong SJ, Serumaga B, et al. The prevalence and nature of prescribing and monitoring errors in English general practice: a retrospective case note review. Brit J General Pract. 2013;63(613):e543-e53.

9. Galbraith A, Bullock S, Manias E, Hunt B, Richards A. Fundamentals of pharmacology: an applied approach for nursing and health. Routledge: London; 2015.

10. Amato, Mary G, et al. Computerized prescriber order entry-related patient safety reports: analysis of 2522 medication errors. J Am Med Inform Assoc. 2017;24(2):316-22.

11. Paterno MD, Maviglia SM, Gorman PN, Seger DL, Yoshida E, Seger AC, et al. Tiering drug-drug interaction alerts by severity increases compliance rates. J Am Med Informatics Assoc. 2009;16(1):40-6.

12. Lewis PJ, Dornan T, Taylor D, Tully MP, Wass V, Ashcroft DM. Prevalence, incidence and nature of prescribing errors in hospital inpatients. Drug Saf. 2009:32(5):379-89.

13. Ahmed B, Nanji K, Mujeeb R, Patel MJ. Effects of polypharmacy on adverse drug reactions among geriatric outpatients at a tertiary care hospital in Karachi: a prospective cohort study. PloS one. 2014;9(11):e112133-e.
14. Opondo D, Eslami S, Visscher S, de Rooij SE, Verheij R, Korevaar JC, et al. Inappropriateness of medication prescriptions to elderly patients in the primary care setting: a systematic review. PloS one. 2012;7(8):e43617-e.

15. Salmasi S, Khan TM, Hong YH, Ming LC, Wong TW. Medication errors in the southeast Asian countries: a systematic review. PLoS One. 2015;10(9):e0136545.

16. Black AD, Car J, Pagliari C, Anandan C, Cresswell K, Bokun T, et al. The impact of eHealth on the quality and safety of health care: a systematic overview. PLoS Med. 2011;8(1):e1000387.

17. Mekonnen AB, McLachlan AJ, Brien J-a E. Pharmacy-led medication reconciliation programmes at hospital transitions: a systematic review and meta-analysis. J Clin Pharm Ther. 2016;41(2):128-44.

18. Chisholm-Burns MA, Lee JK, Spivey CA, Slack M, Herrier RN, Hall-Lipsy E, Zivin JG, et al. US pharmacists' effect as team members on patient care: systematic review and meta-analyses. Med Care. 2010:923-933.

19. Christensen M, Lundh A. Medication review in hospitalised patients to reduce morbidity and mortality. Cochrane Database Syst Rev. 2016;(2). Art. No.: CD008986. https://doi.org/10.1002/14651858.CD008986.pub3.

20. Kim J, Bates DW. Medication administration errors by nurses: adherence to guidelines. J Clin Nurs. 2013;22(3-4):590-8.

21. Khalil H, Bell B, Chambers H, Sheikh A, Avery AJ. Professional, structural and organisational interventions in primary care for reducing medication errors. Cochrane Database Syst Rev. 2017;10(10):CD003942. Published 2017 Oct 4. https://doi.org/10.1002/14651858.CD003942.pub3.

22. Sears K, Ross-White A, Godfrey CM. The incidence, prevalence and contributing factors associated with the occurrence of medication errors for children and adults in the community setting: A systematic review. JB Database Syst Rev Implement Rep. 2012;10(35):2350-464.

23. Khalil H, Kirschbaum M. Adverse drug reactions in primary care: a scoping review protocol. JBI Database System Rev Implement Rep. 2018;16(3):615-21.

24. Khalil H, Peters M, Godfrey CM, McInerney P, Soares CB, Parker D. An evidence-based approach to scoping reviews. Worldviews Evid-Based Nurs. 2016;13(2):118-23

25. Peters MD, Godfrey CM, Khalil H, Mclnerney P, Parker D, Soares CB. Guidance for conducting systematic scoping reviews. Int J Evidence-based Healthcare. 2015;13(3):141-6.

26. Gandhi TK, Weingart SN, Borus J, Seger AC, Peterson J, Burdick E, et al. Adverse drug events in ambulatory care. N Engl J Med. 2003;348(16): 1556-64.

27. Gurwitz JH, Field TS, Judge J, Rochon P, Harrold LR, Cadoret C, et al. The incidence of adverse drug events in two large academic long-term care facilities. Am J Med. 2005;118(3):251-8.

28. Hakkarainen KM, Gyllensten H, Jonsson AK, Andersson Sundell K, Petzold M, Hagg S. Prevalence, nature and potential preventability of adverse drug events - a population-based medical record study of 4970 adults. Br J Clin Pharmacol. 2014;78(1):170-83.

29. Jacobs TQ, Ross A. Adverse effects profile of multidrug-resistant tuberculosis treatment in a south African outpatient clinic. S Afr Fam Pract. 2012;54(6):531-9.

30. Kaushal R, Goldmann DA, Keohane CA, Christino M, Honour M, Hale AS, et al. Adverse drug events in pediatric outpatients. Ambul Pediatr. 2007; 7(5):383-9.

31. Kowski AB, Weissinger F, Gaus V, Fidzinski P, Losch F, Holtkamp M. Specific adverse effects of antiepileptic drugs--a true-to-life monotherapy study. Epilepsy Behav. 2016:54:150-7.

32. Lahon KI, Shetty HM, Paramel AM, Sharma GY. Adverse drug reaction monitoring of antipsychotics, antidepressants and mood stabilizers in the psychiatric outpatient unit of a teaching hospital-a retrospective study. Int J Pharma Bio Sci. 2012;3:470-8.

33. Lin HY, Liao CC, Cheng SH, Wang PC, Hsueh YS. Association of potentially inappropriate medication use with adverse outcomes in ambulatory elderly patients with chronic diseases: experience in a Taiwanese medical setting. Drugs Aging. 2008;25(1):49-59 Bio Sciences 2012;3(1):P470-P8.

34. Li BK, Tian EAL. Adverse drug reactions to oral antibiotics used for dermatological indications: A preliminary study. Ann Acad Med Singapore. 2014;1:S257.

35. Milligan F. Diabetes medication incidents in the care home setting. Nurs Stand. 2012;26(29):38-43

36. Montserrat-Capella D, Suarez M, Ortiz L, Mira JJ, Duarte HG, Reveiz L, et al. Frequency of ambulatory care adverse events in Latin American countries: the AMBEAS/PAHO cohort study. Int I Qual Health Care. 2015;27(1):52-9. 
37. Rosales Z, Leon L, Gomez-Gomez A, Arietti L, Cour EP, Rueda JLF, et al. Adverse events to biologic agents in elderly patients with rheumatoid arthritis: Cohort with 13 years of follow-up. Arthritis and Rheumatology. Conference: American College of Rheumatology/Association of Rheumatology Health Professionals Annual Scientific Meeting, ACR/ARHP 2015;67(no pagination).

38. Schildmeijer KGI, Unbeck M, Ekstedt M, Lindblad M, Nilsson L. Adverse events in patients in home healthcare: a retrospective record review using trigger tool methodology. BMJ Open. 2018;8(1):e019267.

39. Shehab N, Patel PR, Srinivasan A, Budnitz DS. Emergency department visits for antibiotic-associated adverse events. Clin Infect Dis. 2008;47(6):735-43.

40. Theitler J, Berkovitch M, Gandelman-Marton R. Antiepileptic drug treatment in elderly patients with epilepsy. Epilepsia. 2016;57:65.

41. Tomlin A, Reith D, Dovey S, Tilyard M. Methods for retrospective detection of drug safety signals and adverse events in electronic general practice records. Drug Saf. 2012;35(9):733-43.

42. Tsang C, Majeed A, Banarsee R, Gnani S, Aylin P. Recording of adverse events in English general practice: analysis of data from electronic patient records. Informatics Primary Care. 2010;18(2):117-24.

43. Woods DM, Thomas EJ, Holl JL, Weiss KB, Brennan TA. Ambulatory care adverse events and preventable adverse events leading to a hospital admission. Quality Safety Health Care. 2007;16(2):127-31.

44. Wucherer D, Thyrian JR, Eichler T, Hertel J, Kilimann I, Richter S, et al. Drugrelated problems in community-dwelling primary care patients screened positive for dementia. Int Psychogeriatr. 2017;29(11):1857-68.

45. lasella $\mathrm{CJ}$, Johnson HJ, Dunn MA. Adverse drug reactions: type a (intrinsic) or type B (idiosyncratic). Clin Liver Disease. 2017;21(1):73-87.

46. Bouvy JC, De Bruin ML, Koopmanschap MA. Epidemiology of adverse drug reactions in Europe: a review of recent observational studies. Drug Saf. 2015; 38(5):437-53.

47. Masotti P, McColl MA, Green M. Adverse events experienced by homecare patients: a scoping review of the literature. Int I Qual Health Care. 2010; 22(2):115-25.

48. Kongkaew C, Noyce PR, Ashcroft DM. Hospital admissions associated with adverse drug reactions: a systematic review of prospective observational studies. Ann Pharmacother. 2008;42(7):1017-25.

49. Al Hamid A, Ghaleb M, Aljadhey H, Aslanpour Z. A systematic review of hospitalization resulting from medicine-related problems in adult patients. Br J Clin Pharmacol. 2013;78(2):202-17.

50. Mouton JP, Mehta U, Parrish AG, Wilson DP, Stewart A, Njuguna CW, Kramer N, Maartens G, Blockman M, Cohen K. Mortality from adverse drug reactions in adult medical inpatients at four hospitals in South Africa: a cross-sectional survey. Brit J Clin Pharmacol. 2015;80(4):818-26.

51. Khalil H, Lee $S$. Medication safety challenges in primary care: nurses' perspective. J Clin Nurs. 2018;27(9-10):2072-82.

52. Lopez-Gonzalez E, Herdeiro MT, Figueiras A. Determinants of underreporting of adverse drug reactions. Drug Saf. 2009;32(1):19-31.

53. Kostoff RN. Under-reporting of adverse events in the biomedical literature. J Data Information Sci. 2016;1 (4):10-32.

54. Ehmann F, Caneva L, Prasad K, Paulmichl M, Maliepaard M, Llerena A, Ingelman-Sundberg M, Papaluca-Amati M. Pharmacogenomic information in drug labels: European medicines agency perspective. Pharmacogenomics J. 2015;15(3):201.

\section{Publisher's Note}

Springer Nature remains neutral with regard to jurisdictional claims in published maps and institutional affiliations.

Ready to submit your research? Choose BMC and benefit from:
- fast, convenient online submission
- thorough peer review by experienced researchers in your field
- rapid publication on acceptance
- support for research data, including large and complex data types
- gold Open Access which fosters wider collaboration and increased citations
- maximum visibility for your research: over 100M website views per year
At BMC, research is always in progress.
Learn more biomedcentral.com/submissions

